\title{
Accurate optical spectra through time-dependent density functional theory based on screening-dependent hybrid functionals
}

\author{
Alexey Tal $\odot,{ }^{1, *}$ Peitao Liu $\odot,{ }^{2}$ Georg Kresse, ${ }^{2}$ and Alfredo Pasquarello $\odot^{1}$ \\ ${ }^{1}$ Chaire de Simulation à l'Echelle Atomique (CSEA), Ecole Polytechnique Fédérale de Lausanne (EPFL), CH-1015 Lausanne, Switzerland \\ ${ }^{2}$ University of Vienna, Faculty of Physics and Center for Computational Materials Science, Sensengasse 8, A-1090 Vienna, Austria
}

(Received 3 February 2020; revised 23 April 2020; accepted 24 June 2020; published 20 July 2020)

\begin{abstract}
We investigate optical absorption spectra obtained through time-dependent density functional theory (TDDFT) based on nonempirical hybrid functionals that are designed to correctly reproduce the dielectric function. The comparison with state-of-the-art $G W$ calculations followed by the solution of the Bethe-Salpeter equation (BSE- $G W$ ) shows close agreement for both the transition energies and the main features of the spectra. We confront TD-DFT with BSE- $G W$ by focusing on the model dielectric function and the local exchange-correlation kernel. The present TD-DFT approach achieves the accuracy of BSE- $G W$ at a fraction of the computational cost.
\end{abstract}

DOI: 10.1103/PhysRevResearch.2.032019

Semilocal density functionals are notoriously unsuitable for describing band gaps in semiconductors due to the lack of a derivative discontinuity [1,2]. Thus, most of the $a b$ initio methods for optical absorption calculations based on density functional theory (DFT) have to address two important problems. First, it is necessary to correct the band gap. Second, the interaction between electrons and holes has to be taken into account. The state-of-the-art approach to improve the band gap is the $G W$ approximation [3-6], whereas the electron-hole interaction can be included by solving the Bethe-Salpeter equation (BSE) [7-11]. The combined BSE-GW approach has been shown to give very accurate results compared to experiment, but the main drawback is the scaling, which makes it computationally very challenging for large size systems.

Gross developed an alternative approach based on the timedependent electron density, typically referred to as the timedependent density functional theory (TD-DFT) [7,12,13]. In this approach, the time-dependent Kohn-Sham equations include a time-dependent exchange-correlation (xc) potential $v_{\mathrm{xc}}$ and its variation with the time-dependent density, also known as the exchange-correlation kernel $f_{\mathrm{xc}}$. The exact $v_{\mathrm{xc}}$ and $f_{\mathrm{xc}}$ are unknown, but several approximations have been introduced. Local approximations to $f_{\mathrm{xc}}$ lack the correct long-wavelength limit, $f_{\mathrm{xc}}(q \rightarrow 0) \propto 1 / q^{2}$, responsible for the correct description of the electron-hole interaction. Therefore, local approximations are unable to capture excitonic effects, but can perform well for metallic systems [14-17]. The correct asymptotic behavior is recovered in the socalled "nanoquanta" kernel [18-20] derived from the BSE to

\footnotetext{
*alexey.tal@epfl.ch; alyxthal@gmail.com

Published by the American Physical Society under the terms of the Creative Commons Attribution 4.0 International license. Further distribution of this work must maintain attribution to the author(s) and the published article's title, journal citation, and DOI.
}

capture excitonic effects. Hence this approach produces accurate spectra for solids, but remains computationally as expensive as solving the BSE.

Hybrid-functional calculations with a nonlocal Fock exchange can be used to improve the band gaps. Moreover, since the long-wavelength limit is accounted for, it can be expected that these functionals could be used for calculating optical spectra. Various hybrid functionals have been tested in TDDFT and it has been shown that a good performance can be achieved in molecules [21,22]. However, a good description in solids requires the consideration of the screening in the exchange interaction $[23,24]$. Such a screened interaction was found to be crucial for the correct description of optical spectra [23,25].

Several different approximations for the screening of the nonlocal exchange interaction have been investigated [26-30]. The results suggest that hybrid functionals yield spectra comparable to BSE- $G W$ provided the adopted fraction of Fock exchange accounts for the screening in the long range. In particular, Wing et al. obtained good results with screened range-separated hybrid functionals [29]. However, the correct screening in the short and medium range was not imposed but rather followed from the empirical setting of the hybridfunctional parameters in their TD-DFT approach. For instance, their choice of taking $25 \%$ of Fock exchange in the short range does not describe the physically correct behavior of the screening. More importantly, the range separation parameter was empirically tuned so that the calculated band gaps matched the $G W$ ones.

Recently, Chen et al. [31] developed a nonempirical hybrid-functional scheme, in which all the parameters are taken from the static screening without tuning. The method showed very accurate electronic structures and band gaps for a large number of semiconductors and insulators. The advantage of this approach is that it accurately accounts for the wave-vector-dependent screening: At short range the exchange interaction is only weakly screened, whereas in the long range it is reduced by the static dielectric constant. 
In this Rapid Communication, we investigate the performance of hybrid-functional TD-DFT for optical absorption calculations through a comparison with state-of-the-art BSE$G W$. In the TD-DFT scheme, we employ hybrid functionals that have been designed to reproduce the correct screening properties through a self-consistent procedure [31]. We show that this scheme provides absorption spectra with an accuracy comparable to that of BSE- $G W$ without tuning parameters. In particular, we show that equivalent descriptions of the screening in TD-DFT and BSE- $G W$ result in very similar absorption spectra.

Following recent work on dielectric-dependent hybrid functionals (DDH) [31-33], we use in this work the explicit form of the exchange-correlation potential given by

$$
\begin{aligned}
V_{\mathrm{xc}}\left(\mathbf{r}, \mathbf{r}^{\prime}\right)= & {\left[1-\left(1-\epsilon_{\infty}^{-1}\right) \operatorname{erf}\left(\mu\left|\mathbf{r}-\mathbf{r}^{\prime}\right|\right)\right] V_{\mathrm{x}}^{\mathrm{Fock}}\left(\mathbf{r}, \mathbf{r}^{\prime}\right) } \\
& +\left(1-\epsilon_{\infty}^{-1}\right) V_{\mathrm{x}}^{\mathrm{PBE}, \mathrm{LR}}(\mathbf{r} ; \mu) \delta\left(\mathbf{r}-\mathbf{r}^{\prime}\right) \\
& +V_{\mathrm{c}}^{\mathrm{PBE}}(\mathbf{r}) \delta\left(\mathbf{r}-\mathbf{r}^{\prime}\right),
\end{aligned}
$$

where $\mu$ is the range-separation parameter, and $V_{\mathrm{x}}^{\mathrm{PBE}}$ and $V_{\mathrm{c}}^{\mathrm{PBE}}$ are the Perdew-Burke-Ernzerhof (PBE) exchange and correlation potentials [34]. Here, $V_{\mathrm{x}}^{\text {Fock }}$ is the Fock exchange operator given by

$$
V_{\mathrm{x}}^{\text {Fock }}\left(\mathbf{r}, \mathbf{r}^{\prime}\right)=-e^{2} \frac{1}{N_{\mathbf{k}}} \sum_{n \mathbf{k}} \frac{\psi_{n \mathbf{k}}^{*}\left(\mathbf{r}^{\prime}\right) \psi_{n \mathbf{k}}(\mathbf{r})}{\left|\mathbf{r}-\mathbf{r}^{\prime}\right|}
$$

where $\psi_{n \mathbf{k}}$ are one-electron Bloch states, the sum over $\mathbf{k}$ is over $N_{\mathbf{k}} \mathbf{k}$ points of the Brillouin zone, and the sum over $n$ is over all the occupied bands. In Eq. (1), the Fock exchange interaction in reciprocal space is thus multiplied by the function [33]

$$
c_{\mathrm{x}}^{\mathrm{DDH}}(|\mathbf{q}+\mathbf{G}|)=1-\left(1-\epsilon_{\infty}^{-1}\right) e^{-|\mathbf{q}+\mathbf{G}|^{2} / 4 \mu^{2}} .
$$

In the $G W$ approximation, the Coulomb interaction is screened by the dielectric function which is a frequencydependent tensor $\epsilon_{\mathbf{G}, \mathbf{G}^{\prime}}^{-1}(\mathbf{q}, \omega)$ [6]. Thus, $c_{\mathrm{x}}^{\mathrm{DDH}}(|\mathbf{q}+\mathbf{G}|)$ in Eq. (3) corresponds to a model inverse dielectric function $\epsilon_{\text {model }}^{-1}(|\mathbf{q}+\mathbf{G}|)$ that neglects the dynamical screening $(\omega \neq 0)$ and the off-diagonal elements.

In the approach of Ref. [31], the parameters in Eq. (3) are determined self-consistently. In the long-wavelength limit, the interaction is set to $1 /\left(\epsilon_{\infty} q^{2}\right)$, where the dielectric constant $\epsilon_{\infty}$ is calculated using the random-phase approximation with vertex corrections. The parameter $\mu$ is obtained by fitting the model to the calculated dielectric function. In Fig. 1, the model dielectric functions associated with the DDH are compared to the diagonal elements of the dielectric matrix at the $\Gamma$ point at zero frequency. The dielectric matrix is obtained within partially self-consistent $G W$ with vertex corrections [cf. Supplemental Material (SM) [35]]. In all the cases, we find the model dielectric function to be in good agreement with the calculated dielectric function.

The excitation spectra in both BSE and TD-DFT are obtained by solving an eigenvalue problem, referred to as the Bethe-Salpeter and Casida equation, respectively $[7,36]$,

$$
\left(\begin{array}{cc}
\mathbf{A} & \mathbf{B} \\
\mathbf{B}^{*} & \mathbf{A}^{*}
\end{array}\right)\left(\begin{array}{l}
\mathbf{X} \\
\mathbf{Y}
\end{array}\right)=\Omega\left(\begin{array}{cc}
\mathbb{1} & 0 \\
0 & -\mathbb{1}
\end{array}\right)\left(\begin{array}{l}
\mathbf{X} \\
\mathbf{Y}
\end{array}\right)
$$
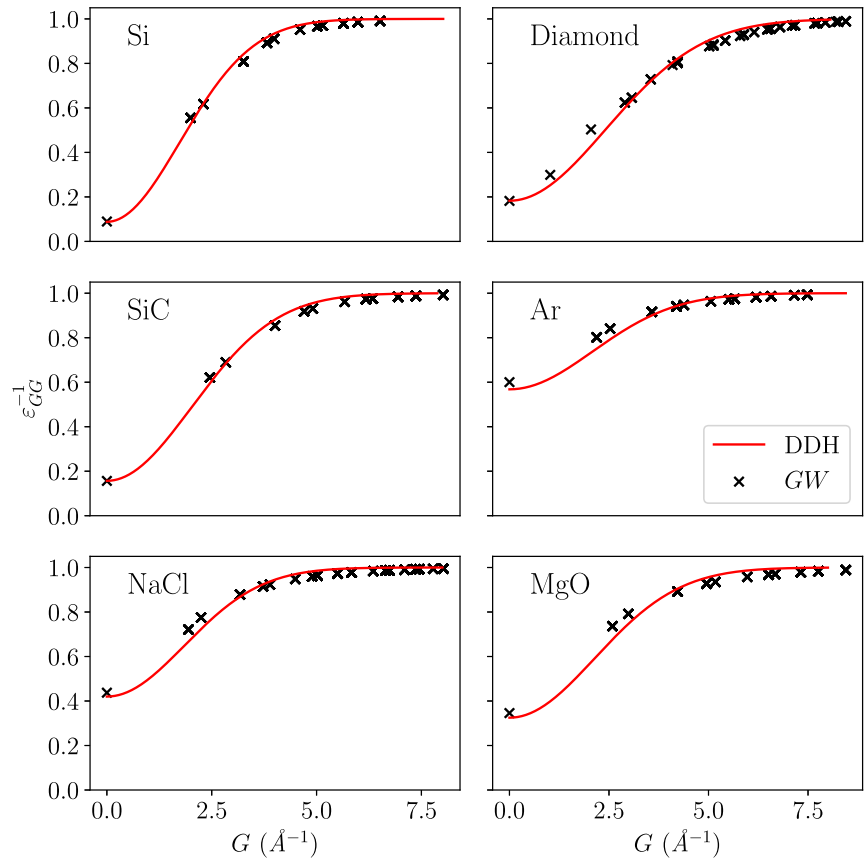

FIG. 1. Inverse dielectric functions vs wave vector at the $\Gamma$ point for $\mathrm{Si}$, diamond, $\mathrm{SiC}, \mathrm{Ar}, \mathrm{NaCl}$, and $\mathrm{MgO}$, as calculated in $G W$ and given by the model in Eq. (3) with parameters taken from Ref. [31].

where submatrices $\mathbf{A}$ and $\mathbf{B}$ read

$$
\begin{gathered}
A_{a i, b j}=\left(\epsilon_{a}-\epsilon_{i}\right) \delta_{i, j} \delta_{a, b}+\langle i b|K| a j\rangle, \\
B_{a i, b j}=\langle i j|K| a b\rangle,
\end{gathered}
$$

with the indices $i, j$ and $a, b$ referring to occupied and unoccupied states, respectively. The excitation frequencies of the system are given by $\Omega . X$ and $Y$ are the two-body electron-hole eigenstates in the transition basis $\psi_{a}(\mathbf{r}) \psi_{i}^{*}\left(\mathbf{r}^{\prime}\right)$ and $\psi_{i}(\mathbf{r}) \psi_{a}^{*}\left(\mathbf{r}^{\prime}\right)$. Matrix $A$ includes two terms, the energy of the direct transition from occupied to unoccupied states and the electron-hole interaction described by the kernel $K$ [37,38]. Equation (4) is non-Hermitian, which makes it difficult to solve with standard eigenvalue solvers $[39,40]$. A common practice to avoid this difficulty is to neglect the coupling between excitations and deexcitations by setting $B$ to zero. This approximation is known as the Tamm-Dancoff approximation.

The distinction between BSE-GW and TD-DFT approaches results, on the one hand, from the origin of the one-particle eigenfunctions and energies and, on the other hand, from the type of interaction kernel $K$. To make the comparison between different methods more transparent, we provide in Fig. 2 the Feynman diagrams corresponding to the various irreducible polarizabilities $\tilde{\chi}$ discussed in this work.

In BSE- $G W$, the orbitals and energies are derived from a preceding $G W$ calculation and the kernel consists of a Hartree term $V$ and a screened exchange term $W$ [38],

$$
\langle i b|K| a j\rangle=2\langle i b|V| a j\rangle-\langle i b|W| j a\rangle .
$$

The Hartree term describes the bare Coulomb interaction and is the same in all the approximations considered here. It can be included straightforwardly in a two-point formulation 


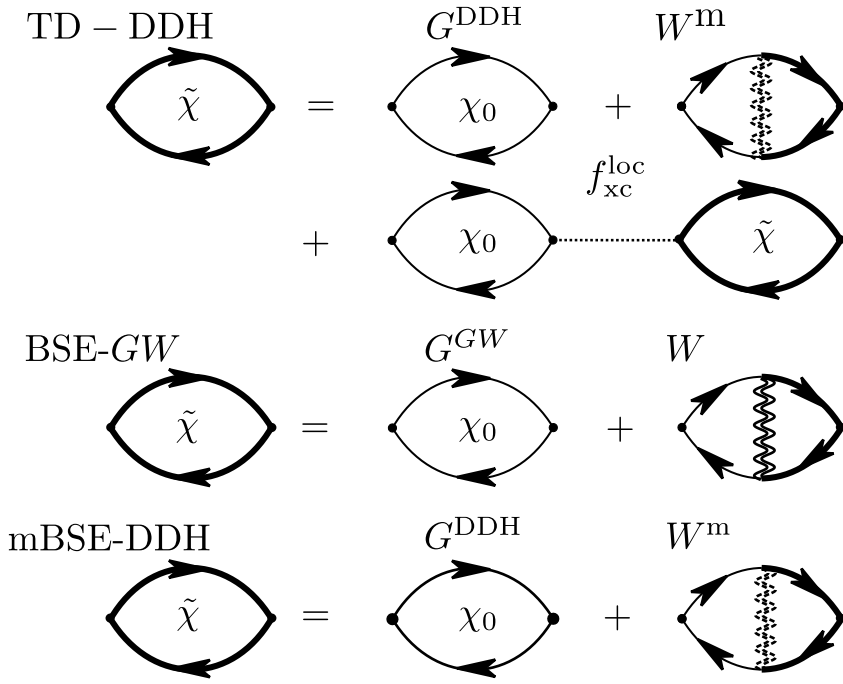

FIG. 2. Irreducible polarizabilities $\tilde{\chi}$ in various approximations. The reducible polarizability is obtained from $\chi=\tilde{\chi}+\tilde{\chi} v \chi$. The wiggly line indicates the screened interaction $W$.

involving the polarizability $\chi$. The exchange term, however, requires calculating four-point integrals, which drastically increases the complexity of the problem. The screening of the exchange interaction is determined by the frequencydependent dielectric function $\epsilon$ obtained from $G W$ and is represented by a vertical wiggly line in the diagrams. However, as shown in Refs. [41-43], the dynamical effects can often be neglected in BSE calculations.

In the TD-DFT approach, the electron energies and wave functions are obtained from a semilocal or hybrid-functional calculation. The interaction kernel consists of three terms, a Hartree and a screened exchange term $W^{\mathrm{m}}$ as in the BSE, and an additional local exchange-correlation interaction $f_{\mathrm{xc}}^{\text {loc }}$ [44],

$$
\langle i b|K| a j\rangle=2\langle i b|V| a j\rangle-\left\langle i b\left|W^{\mathrm{m}}\right| j a\right\rangle+\left\langle i b\left|f_{\mathrm{xc}}^{\text {loc }}\right| a j\right\rangle .
$$

The screening of the exchange interaction in TD-DFT is described by a constant through $W^{\mathrm{m}}=c_{\mathrm{x}} V(|\mathbf{q}+\mathbf{G}|)$ or by a function through $W^{\mathrm{m}}=c_{\mathrm{x}}(|\mathbf{q}+\mathbf{G}|) V(|\mathbf{q}+\mathbf{G}|)$, depending on the exchange-correlation functional. In particular, $c_{\mathrm{x}}=0$ for semilocal DFT functionals. In the case of DDH, the exchange interaction is screened by the model inverse dielectric function $c_{\mathrm{x}}=c_{\mathrm{x}}^{\mathrm{DDH}}(|\mathbf{q}+\mathbf{G}|)$ given in Eq. (3) and the local exchange-correlation interaction $f_{\mathrm{xc}}^{\text {loc }}$ is derived from the local part of the exchange-correlation potential

$$
f_{\mathrm{xc}}^{\mathrm{loc}}\left(\mathbf{r}, \mathbf{r}^{\prime}\right)=\frac{\delta\left\{V_{\mathrm{c}}^{\mathrm{PBE}}+\left(1-\epsilon_{\infty}^{-1}\right) V_{\mathrm{x}}^{\mathrm{PBE}, \mathrm{LR}}\right\}}{\delta \rho(\mathbf{r})} \delta\left(\mathbf{r}-\mathbf{r}^{\prime}\right) .
$$

In Fig. $2, f_{\mathrm{xc}}^{\text {loc }}$ is represented by a dotted line connecting $\chi_{0}$ and $\tilde{\chi}$. In this work, we refer to this version of TD-DFT as TD-DDH.

Next, we focus on the comparison between BSE- $G W$ and TD-DDH. In both schemes, the absorption spectra are obtained from the eigenvalue problem in Eq. (4). In particular, the BSE- $G W$ calculations are based on partially selfconsistent $G W$ using the "nanoquanta" vertex corrections $f_{\mathrm{xc}}$
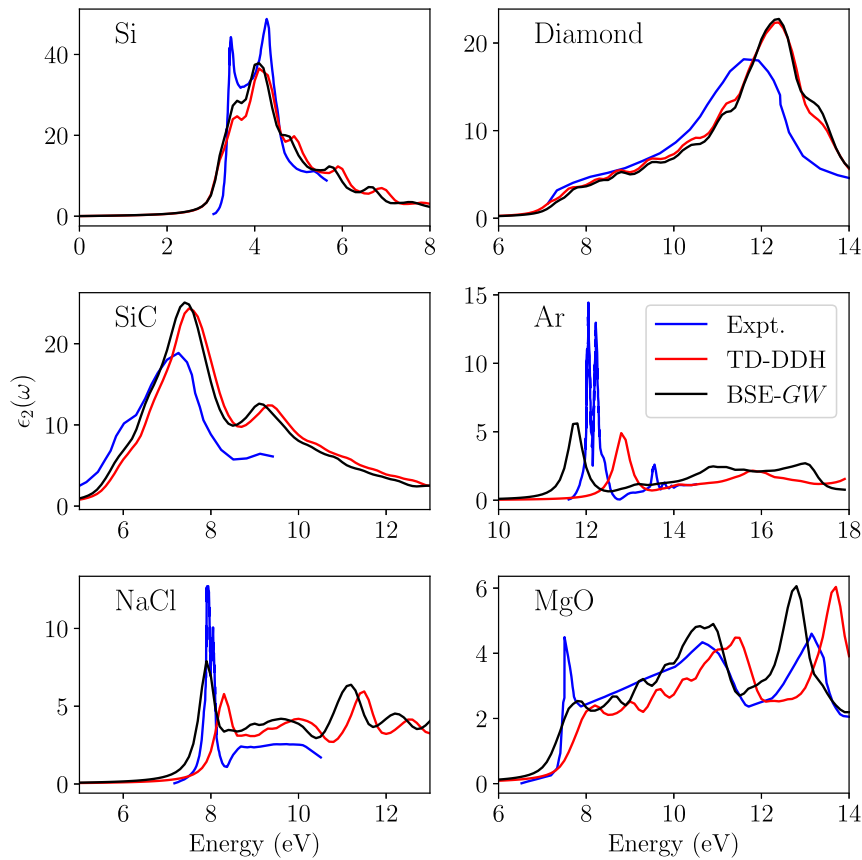

FIG. 3. Absorption spectra for $\mathrm{Si}$, diamond, $\mathrm{SiC}, \mathrm{Ar}, \mathrm{NaCl}$, and $\mathrm{MgO}$ calculated with BSE- $G W$ and TD-DDH. Experimentally measured spectra are taken from Ref. [45] for diamond, from Ref. [46] for $\mathrm{Si}$, from Ref. [47] for $\mathrm{SiC}$, from Ref. [48] for Ar, from Ref. [49] for $\mathrm{NaCl}$, and from Ref. [50] for $\mathrm{MgO}$.

in the polarizability $\tilde{\chi}$ [51]. These two approaches are tested on a set of materials possessing a wide range of band gaps. The corresponding spectra are given in Fig. 3. Our calculations show that both approaches agree well with experiment and that TD-DDH reproduces all the spectral features with the correct oscillator strengths. In the case of diamond, $\mathrm{Si}$, and $\mathrm{SiC}$, the spectra are nearly on top of each other. For $\mathrm{Ar}, \mathrm{NaCl}$, and $\mathrm{MgO}$, the relative positions of the main features in the spectra are found to be shifted slightly. In principle, this shift can result from differences in the band structure and in the screening. Our analysis indicates that the dominant effect is due to the energy transition terms in Eq. (5). From Table I, we

TABLE I. Band gaps (in eV) obtained with the semilocal PBE functional [34], DDH, and $G W$. The experimental values are augmented by theoretical corrections resulting from the coupling to phonons.

\begin{tabular}{lcccccc}
\hline \hline & $\mathrm{Si}$ & $\mathrm{SiC}$ & Diamond & $\mathrm{NaCl}$ & $\mathrm{Ar}$ & $\mathrm{MgO}$ \\
\hline PBE & 0.75 & 1.35 & 4.14 & 5.21 & 8.70 & 4.77 \\
DDH & 1.31 & 2.50 & 5.69 & 9.13 & 14.60 & 8.41 \\
$G W$ & 1.41 & 2.55 & 5.85 & 8.86 & 13.75 & 8.12 \\
Expt. & $1.23^{\mathrm{a}}$ & $2.53^{\mathrm{b}}$ & $5.85^{\mathrm{c}}$ & $9.14^{\mathrm{d}}$ & $14.33^{\mathrm{e}}$ & $8.36^{\mathrm{f}}$ \\
\hline \hline
\end{tabular}

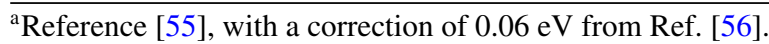

${ }^{\mathrm{b}}$ Reference [57], with a correction of $0.11 \mathrm{eV}$ from Ref. [58].

${ }^{\mathrm{c}}$ Reference [59], with a correction of $0.37 \mathrm{eV}$ from Ref. [56].

${ }^{\mathrm{d}}$ Reference [49], with a correction of $0.17 \mathrm{eV}$ from Ref. [60].

${ }^{\mathrm{e}}$ Reference [61], with a correction of $0.03 \mathrm{eV}$ calculated using the method described in Refs. [62,63].

${ }^{\mathrm{f}}$ Reference [64], with a correction of $0.53 \mathrm{eV}$ from Ref. [65]. 




FIG. 4. Optical absorption spectrum of diamond calculated with BSE and full $\epsilon^{-1}$ from $G W\left(W^{\text {full }}\right)$, BSE and diagonal $\epsilon^{-1}\left(W^{\text {diag }}\right)$, BSE and model $\epsilon_{\text {model }}^{-1}\left(W^{\mathrm{m}}\right)$, and TD-DDH and model $\epsilon_{\text {model }}^{-1}$ with $f_{\mathrm{xc}}^{\text {loc }}$ $\left(W^{\mathrm{m}}+f_{\mathrm{xc}}^{\text {loc }}\right)$. All spectra are based on energies and wave functions from a $G_{0} W_{0}$ calculation. A $6 \times 6 \times 6 \mathbf{k}$-point grid is used.

notice that the calculated band gaps differ by less than $0.15 \mathrm{eV}$ for $\mathrm{Si}, \mathrm{SiC}$, and diamond, but that the disagreement is more substantial for $\mathrm{NaCl}, \mathrm{Ar}$, and $\mathrm{MgO}$. Overall, when compared to experimental values corrected for the coupling to phonons, DDH and $G W$ band gaps show mean average errors of 0.11 and $0.22 \mathrm{eV}$, respectively. These errors are consistent with the current accuracy of $a b$ initio methods [52,53], indicating that the agreement with experiment should be considered excellent for both schemes. As far as the screening is concerned, we show below that the small discrepancies observed in Fig. 1 hardly change the spectra.

The performance of TD-DDH can also be assessed through a comparison with TD-PBE0, an approach commonly used for the calculations of spectra [26,54]. TD-PBE0 is based on a global hybrid functional where $25 \%$ of Fock exchange is used uniformly and the exchange interaction in the calculation of the spectra is screened by $\varepsilon_{\infty}$. As shown in the SM [35], the accuracy of TD-DDH is significantly better. Moreover, TD-PBE0 does not reproduce the dielectric screening over the full range, which obscures the understanding of the underlying physics.

To compare the screening in BSE- $G W$ and TD-DDH, we show in Fig. 4 the absorption spectra of diamond calculated in various approximations using the same energies and wave functions, which are taken from a $G_{0} W_{0}$ calculation. We start our analysis from a BSE calculation in which the full static inverse dielectric matrix is used $\left(W^{\text {full }}\right)$. In particular, we show that the off-diagonal elements of this matrix barely have any effect on the calculated spectrum $\left(W^{\text {diag }}\right)$, in accordance with Ref. [30]. Next, we replace the inverse dielectric function with the model $c_{\mathrm{x}}^{\mathrm{DDH}}(|\mathbf{q}+\mathbf{G}|)$ and find no discernible difference in the spectrum $\left(W^{\mathrm{m}}\right)$. Notice that we here consider isotropic screening and that the extension of this model to anisotropic materials remains to be investigated. The present treatment of the screening is equivalent to that of TD-DDH, where the local exchange-correlation kernel is neglected, i.e., $f_{\mathrm{xc}}^{\text {loc }}=0$, also referred to as model BSE (mBSE) $[30,66]$. To restore the full TD-DDH screening, we include the $f_{\mathrm{xc}}^{\text {loc }}$ and still obtain essentially the same spectrum $\left(W^{\mathrm{m}}+f_{\mathrm{xc}}^{\text {loc }}\right)$. Hence, these results indicate that the model screening in TD-DDH gives an accurate description of the screening in $W=\epsilon^{-1} V$ and that the effect of $f_{\mathrm{xc}}^{\text {loc }}$ is negligible for extended systems. Considering this, we can say that for extended systems TD-DDH is de facto equivalent to mBSE.

The numerical complexity of Eq. (4) is the same in BSE and TD-DDH. However, the preceding $G W$ calculations required in BSE- $G W$ involve a high computational cost, which scales as $N^{4}$ in the number of electrons $N$ in most $G W$ implementations instead of as $N^{3}$ in TD-DFT. Additionally, in the calculation of the Green's function in $G W$, the convergence with respect to the number of unoccupied states and the number of frequency points has to be controlled carefully, which significantly increases the complexity of the calculations. Note that the static dielectric constant only needs to be determined at the $\Gamma$ point of the Brillouin zone and that it converges quickly with respect to the number of included orbitals. Furthermore, the hybrid-functional approach only requires a model static dielectric function, for which the static limit can be obtained rather efficiently [32]. Thus, the hybrid-functional approach opens the way to more efficient numerical schemes that can circumvent the calculation of the full dielectric matrix.

In conclusion, we have shown that time-dependent calculations using the parameter-free DDH functional yield optical absorption spectra with an accuracy comparable to BSE- $G W$. The success of this approach originates from the use of a model dielectric function that gives a physically motivated description of the screened exchange interaction over the full spatial range. Notably, the computational complexity of the method is drastically reduced compared to BSE- $G W$, as it eliminates the need for preceding $G W$ calculations. This will allow one to consider larger and more complex systems than hitherto possible.

The structures and the input files used for the calculations are freely available on the Materials Cloud platform, see Ref. [67].

Support from the Swiss National Science Foundation is acknowledged under Grant No. 200020-172524. The calculations have been performed at the Swiss National Supercomputing Centre (CSCS) (grant under project ID s879) and at SCITAS-EPFL.
[1] J. P. Perdew and M. Levy, Physical Content of the Exact KohnSham Orbital Energies: Band Gaps and Derivative Discontinuities, Phys. Rev. Lett. 51, 1884 (1983).

[2] L. J. Sham and M. Schlüter, Density-Functional Theory of the Energy Gap, Phys. Rev. Lett. 51, 1888 (1983).
[3] L. Hedin, New method for calculating the one-particle Green's function with application to the electron-gas problem, Phys. Rev. 139, A796 (1965).

[4] L. Hedin and S. Lundqvist, Effects of electron-electron and electron-phonon interactions on the one-electron states of 
solids, in Solid State Physics, edited by F. Seitz, D. Turnbull, and H. Ehrenreich (Academic, New York, 1970), Vol. 23, pp. 1-181.

[5] M. S. Hybertsen and S. G. Louie, First-Principles Theory of Quasiparticles: Calculation of Band Gaps in Semiconductors and Insulators, Phys. Rev. Lett. 55, 1418 (1985).

[6] F. Aryasetiawan and O. Gunnarsson, The $G W$ method, Rep. Prog. Phys. 61, 237 (1998).

[7] G. Onida, L. Reining, and A. Rubio, Electronic excitations: Density-functional versus many-body Green's-function approaches, Rev. Mod. Phys. 74, 601 (2002).

[8] S. Albrecht, L. Reining, R. Del Sole, and G. Onida, Ab Initio Calculation of Excitonic Effects in the Optical Spectra of Semiconductors, Phys. Rev. Lett. 80, 4510 (1998).

[9] L. X. Benedict, E. L. Shirley, and R. B. Bohn, Optical Absorption of Insulators and the Electron-Hole Interaction: An Ab Initio Calculation, Phys. Rev. Lett. 80, 4514 (1998).

[10] M. Rohlfing and S. G. Louie, Excitonic Effects and the Optical Absorption Spectrum of Hydrogenated Si Clusters, Phys. Rev. Lett. 80, 3320 (1998).

[11] W. Hanke and L. J. Sham, Many-particle effects in the optical spectrum of a semiconductor, Phys. Rev. B 21, 4656 (1980).

[12] E. K. U. Gross and W. Kohn, Local Density-Functional Theory of Frequency-Dependent Linear Response, Phys. Rev. Lett. 55, 2850 (1985).

[13] E. Runge and E. K. U. Gross, Density-Functional Theory for Time-Dependent Systems, Phys. Rev. Lett. 52, 997 (1984).

[14] M. E. Casida, Time-dependent density functional response theory for molecules, in Recent Advances in Density-Functional Methods, edited by D. P. Chong (World Scientific, Singapore, 1995), Part I.

[15] I. Vasiliev, S. Ögüt, and J. R. Chelikowsky, Ab Initio Excitation Spectra and Collective Electronic Response in Atoms and Clusters, Phys. Rev. Lett. 82, 1919 (1999).

[16] A. Rubio, J. A. Alonso, X. Blase, L. C. Balbás, and S. G. Louie, $A b$ Initio Photoabsorption Spectra and Structures of Small Semiconductor and Metal Clusters, Phys. Rev. Lett. 77, 247 (1996).

[17] V. I. Gavrilenko and F. Bechstedt, Local-field and exchangecorrelation effects in optical spectra of semiconductors, Phys. Rev. B 54, 13416 (1996).

[18] F. Sottile, V. Olevano, and L. Reining, Parameter-free Calculation of Response Functions in Time-Dependent DensityFunctional Theory, Phys. Rev. Lett. 91, 056402 (2003).

[19] A. Marini, R. Del Sole, and A. Rubio, Bound Excitons in TimeDependent Density-Functional Theory: Optical and EnergyLoss Spectra, Phys. Rev. Lett. 91, 256402 (2003).

[20] G. Adragna, R. Del Sole, and A. Marini, Ab initio calculation of the exchange-correlation kernel in extended systems, Phys. Rev. B 68, 165108 (2003).

[21] U. Salzner, J. B. Lagowski, P. G. Pickup, and R. A. Poirier, Design of low band gap polymers employing density functional theory - Hybrid functionals ameliorate band gap problem, J. Comput. Chem. 18, 1943 (1997).

[22] A. D. Laurent and D. Jacquemin, TD-DFT benchmarks: A review, Int. J. Quantum Chem. 113, 2019 (2013).

[23] F. Bruneval, F. Sottile, V. Olevano, and L. Reining, Beyond time-dependent exact exchange: The need for long-range correlation, J. Chem. Phys. 124, 144113 (2006).
[24] S. Botti, A. Schindlmayr, R. Del Sole, and L. Reining, Timedependent density-functional theory for extended systems, Rep. Prog. Phys. 70, 357 (2007).

[25] J. Paier, M. Marsman, and G. Kresse, Dielectric properties and excitons for extended systems from hybrid functionals, Phys. Rev. B 78, 121201(R) (2008).

[26] Z.-H. Yang, F. Sottile, and C. A. Ullrich, Simple screened exactexchange approach for excitonic properties in solids, Phys. Rev. B 92, 035202(R) (2015).

[27] S. Refaely-Abramson, M. Jain, S. Sharifzadeh, J. B. Neaton, and L. Kronik, Solid-state optical absorption from optimally tuned time-dependent range-separated hybrid density functional theory, Phys. Rev. B 92, 081204(R) (2015).

[28] J. D. Elliott, N. Colonna, M. Marsili, N. Marzari, and P. Umari, Koopmans meets Bethe-Salpeter: Excitonic optical spectra without $G W$, J. Chem. Theory Comput. 15, 3710 (2019).

[29] D. Wing, J. B. Haber, R. Noff, B. Barker, D. A. Egger, A. Ramasubramaniam, S. G. Louie, J. B. Neaton, and L. Kronik, Comparing time-dependent density functional theory with many-body perturbation theory for semiconductors: Screened range-separated hybrids and the $G W$ plus BetheSalpeter approach, Phys. Rev. Mater. 3, 064603 (2019).

[30] J. Sun, J. Yang, and C. A. Ullrich, Low-cost alternatives to the Bethe-Salpeter equation: Towards simple hybrid functionals for excitonic effects in solids, Phys. Rev. Research 2, 013091 (2020).

[31] W. Chen, G. Miceli, G. M. Rignanese, and A. Pasquarello, Nonempirical dielectric-dependent hybrid functional with range separation for semiconductors and insulators, Phys. Rev. Mater. 2, 073803 (2018).

[32] Z. H. Cui, Y. C. Wang, M. Y. Zhang, X. Xu, and H. Jiang, Doubly screened hybrid functional: An accurate first-principles approach for both narrow- and wide-gap semiconductors, J. Phys. Chem. Lett. 9, 2338 (2018).

[33] P. Liu, C. Franchini, M. Marsman, and G. Kresse, Assessing model-dielectric-dependent hybrid functionals on the antiferromagnetic transition-metal monoxides $\mathrm{MnO}, \mathrm{FeO}, \mathrm{CoO}$, and NiO, J. Phys.: Condens. Matter 32, 015502 (2020).

[34] J. P. Perdew, K. Burke, and M. Ernzerhof, Generalized Gradient Approximation Made Simple, Phys. Rev. Lett. 77, 3865 (1996).

[35] See Supplemental Material at http://link.aps.org/supplemental/ 10.1103/PhysRevResearch.2.032019 for computational details, quasiparticle self-consistent $G W(\mathrm{QS} G W)$, and TD-PBE0 calculations.

[36] T. Sander and G. Kresse, Macroscopic dielectric function within time-dependent density functional theory - Real time evolution versus the Casida approach, J. Chem. Phys. 146, 064110 (2017).

[37] G. Strinati, Effects of dynamical screening on resonances at inner-shell thresholds in semiconductors, Phys. Rev. B 29, 5718 (1984).

[38] M. Rohlfing and S. G. Louie, Electron-Hole Excitations in Semiconductors and Insulators, Phys. Rev. Lett. 81, 2312 (1998).

[39] T. Sander, E. Maggio, and G. Kresse, Beyond the TammDancoff approximation for extended systems using exact diagonalization, Phys. Rev. B 92, 045209 (2015).

[40] E. Maggio and G. Kresse, Correlation energy for the homogeneous electron gas: Exact Bethe-Salpeter solution 
and an approximate evaluation, Phys. Rev. B 93, 235113 (2016).

[41] M. Rohlfing and S. G. Louie, Electron-hole excitations and optical spectra from first principles, Phys. Rev. B 62, 4927 (2000).

[42] F. Bechstedt, K. Tenelsen, B. Adolph, and R. Del Sole, Compensation of Dynamical Quasiparticle and Vertex Corrections in Optical Spectra, Phys. Rev. Lett. 78, 1528 (1997).

[43] A. Marini and R. Del Sole, Dynamical Excitonic Effects in Metals and Semiconductors, Phys. Rev. Lett. 91, 176402 (2003).

[44] M. Casida and M. Huix-Rotllant, Progress in time-dependent density-functional theory, Annu. Rev. Phys. Chem. 63, 287 (2012).

[45] E. D. Palik, Handbook of Optical Constants of Solids, Vol. 1 (Academic, New York, 2012), pp. 1-804.

[46] S. Logothetidis, P. Lautenschlager, and M. Cardona, Temperature dependence of the dielectric function and the interband critical points in orthorhombic GeS, Phys. Rev. B 33, 1110 (1986).

[47] S. Logothetidis and J. Petalas, Dielectric function and reflectivity of 3C-silicon carbide and the component perpendicular to the $c$ axis of $6 \mathrm{H}$-silicon carbide in the energy region $1.5-9.5 \mathrm{eV}$, J. Appl. Phys. 80, 1768 (1996).

[48] V. Saile, M. Skibowski, W. Steinmann, P. Gürtler, E. E. Koch, and A. Kozevnikov, Observation of Surface Excitons in RareGas Solids, Phys. Rev. Lett. 37, 305 (1976).

[49] D. M. Roessler and W. C. Walker, Electronic spectra of crystalline $\mathrm{NaCl}$ and $\mathrm{KCl}$, Phys. Rev. 166, 599 (1968).

[50] M. L. Bortz, R. H. French, D. J. Jones, R. V. Kasowski, and F. S. Ohuchi, Temperature dependence of the electronic structure of oxides: $\mathrm{MgO}, \mathrm{MgAl}_{2} \mathrm{O}_{4}$ and $\mathrm{Al}_{2} \mathrm{O}_{3}$, Phys. Scr. 41, 537 (1990).

[51] Fully self-consistent $G W$ as in QS $G W$ does not lead to appreciable differences in the spectra [35].

[52] M. Shishkin, M. Marsman, and G. Kresse, Accurate Quasiparticle Spectra from Self-Consistent $G W$ Calculations with Vertex Corrections, Phys. Rev. Lett. 99, 246403 (2007).

[53] W. Chen and A. Pasquarello, Accurate band gaps of extended systems via efficient vertex corrections in $G W$, Phys. Rev. B 92, 041115(R) (2015).
[54] M. Vörös and A. Gali, Optical absorption of diamond nanocrystals from ab initio density-functional calculations, Phys. Rev. B 80, 161411(R) (2009).

[55] W. Bludau, A. Onton, and W. Heinke, Temperature dependence of the band gap of silicon, J. Appl. Phys. 45, 1846 (1974).

[56] M. Cardona and M. L. Thewalt, Isotope effects on the optical spectra of semiconductors, Rev. Mod. Phys. 77, 1173 (2005).

[57] R. G. Humphreys, D. Bimberg, and W. J. Choyke, Wavelength modulated absorption in SiC, Solid State Commun. 39, 163 (1981).

[58] B. Monserrat and R. J. Needs, Comparing electron-phonon coupling strength in diamond, silicon, and silicon carbide: Firstprinciples study, Phys. Rev. B 89, 214304 (2014).

[59] C. D. Clark, P. J. Dean, P. V. Harris, and W. C. Price, Intrinsic edge absorption in diamond, Proc. R. Soc. London, Ser. A 277, 312 (1964).

[60] W. R. L. Lambrecht, C. Bhandari, and M. van Schilfgaarde, Lattice polarization effects on the screened Coulomb interaction $W$ of the $G W$ approximation, Phys. Rev. Mater. 1, 43802 (2017).

[61] G. Baldini, Ultraviolet absorption of solid argon, krypton, and xenon, Phys. Rev. 128, 1562 (1962).

[62] M. Zacharias and F. Giustino, One-shot calculation of temperature-dependent optical spectra and phonon-induced band-gap renormalization, Phys. Rev. B 94, 075125 (2016).

[63] F. Karsai, M. Engel, E. Flage-Larsen, and G. Kresse, Electronphonon coupling in semiconductors within the $G W$ approximation, New J. Phys. 20, 123008 (2018).

[64] Y. Hinuma, A. Grüneis, G. Kresse, and F. Oba, Band alignment of semiconductors from density-functional theory and manybody perturbation theory, Phys. Rev. B 90, 155405 (2014).

[65] J. P. Nery, P. B. Allen, G. Antonius, L. Reining, A. Miglio, and X. Gonze, Quasiparticles and phonon satellites in spectral functions of semiconductors and insulators: Cumulants applied to the full first-principles theory and the Fröhlich polaron, Phys. Rev. B 97, 115145 (2018).

[66] M. Bokdam, T. Sander, A. Stroppa, S. Picozzi, D. D. Sarma, C. Franchini, and G. Kresse, Role of polar phonons in the photo excited state of metal halide perovskites, Sci. Rep. 6, 28618 (2016).

[67] 10.24435/materialscloud:gn-2p. 